\title{
Skip Entry of a Capsule-type Manned Space Return Vehicle with Super-orbital Velocity
}

\author{
By Tetsuya YAmAdA ${ }^{1)}$, Isao KAwANO ${ }^{2)}$, Nobutaka KoBAYASHI ${ }^{1), 3), 4)}$ and Seiya Ueno ${ }^{5)}$ \\ ${ }^{1)}$ Div. for Space Flight Engineering , ISAS/JAXA, Sagamihara, Japan \\ ${ }^{2)}$ Aerospace Research and Development Directorate, JAXA, Tsukuba, Japan \\ 3) Graduate Student, Yokohama National University, Yokohama, Japan \\ 4) Present affiliation: NEC, Fuchu, Japan \\ 5) Faculty of Environment and Information Sciences, Yokohama National University, Yokohama, Japan
}

(Received June 24th, 2013)

\begin{abstract}
A capsule-type vehicle is promising for the manned return vehicle from the Moon, Mars or from further bodies from the stand point of the weight budget regardless of its drawback of relatively low lift-to-drag (L/D) aerodynamic characteristics. The present paper describes the 6 degree of freedom (6DoF) simulation of the skip entry of the 4-ton class manned space return vehicle and discussed the issues related to the RCS bank control : the characteristics of the undesirable attitude motion due to leakage of the control force. Moreover, a fault tolerance algorithm based on extemded Kalman-filter (EKF) and the Sequential Monte Calro (SMC) has been introduced and applied to the state estimation in case that the roll rate signal and GPS signals are lost in the GPS/IMU integrated navigation system. The numerical simulation has showed that EKF+SMC fault tolerance algorithm successfully achieved state estimation of vehicle flight positioning by integrating IMU signal even under the condition that the roll rate and GPS signals are lost.
\end{abstract}

Key Words : Skip Entry, Manned Space Flight, Super-orbital Velocity, Reentry Vehicle

\section{Introduction}

Hayabusa-1 SRC successfully returned to the earth in 2010, and post flight analyses were carried out for the sake of future reentry missions ${ }^{1)}$. The technologies regarding to unmanned small reentry capsule was well-demonstrated and the technologies will be succeeded to the further sample return missions ${ }^{2)}$. While, the key technology required for the manned return vehicle contains not a small amount of different issues; Especially as for the issues on return with super-orbital velocity, need to be considered are 1) relaxation of the reentry environment, 2) to enlarge the return corridor and the window (period and landing site) 3) and to achieve the above with secure reliability. Skip entry is important technology in reentry with super-orbital velocity because it can potentially relax the flight environment, and enlarge the down range distance, which is advantageous for the landing site selection regardless of the population density of the site, climate at the reentry date etc.

A capsule-type vehicle is promising for the return from the Moon, Mars or from further bodies from the stand point of the weight budget regardless of its drawback of low liftto-drag (L/D) ratio of 0.3 at maximum. Vehicle flight control needs to be carried out through bank-control by reaction control systems (RCS) which is forced to be asymmetrically arranged due to the aft-body aerodynamic heating. The asymmetrical arrangement causes the undesirable attitude motion of the vehicle, as leakage of bank-control forces to the forces around pitch and yaw axis.
The bank control errors tend to decrease the L/D control efficiency and may lead to errors in guidance in landing point.The present paper describes the 6 degree of freedom (6DoF) simulation of the skip entry of the 4-ton class vehicle and discussed the related issues.

One of the significant feature of the guidance and control of the manned space vehicle is the fault tolerance. Historically, two-fault tolerance (2FT) to protect against loss of the crew has been taken for granted in human space flight vehicle design. From a navigation perspective, one of the most robust means of achieving this goal is to compare the outputs of several independent, dissimilar, but equally performing sensors and then use a center select algorithm. Because significant abnormal data errors often fail to accomplish 2FT in the center select algorithm, the coincided 2 data out of over 4 navigation data are to be used for the judgement after the mutual comparison. This design can tolerate two simultaneous or systematic failures, including common cause failures, and thus fault detection, isolation and recovery of functions all occur automatically with no delay or disruption. In order to increase the failure tolerance, an example of the state value estimation method different to "decision by majority" nor "center-select algorithm" is introduced in the present paper.

In guidance, signal quality of the sensor output signal also affect on the accuracy of guidance and navigation. Effect of the signal quality (noise) and errors of the bankangle and the rate sensors are investigated qualitatively and we try to estimate state even in case of signal loss, and to 


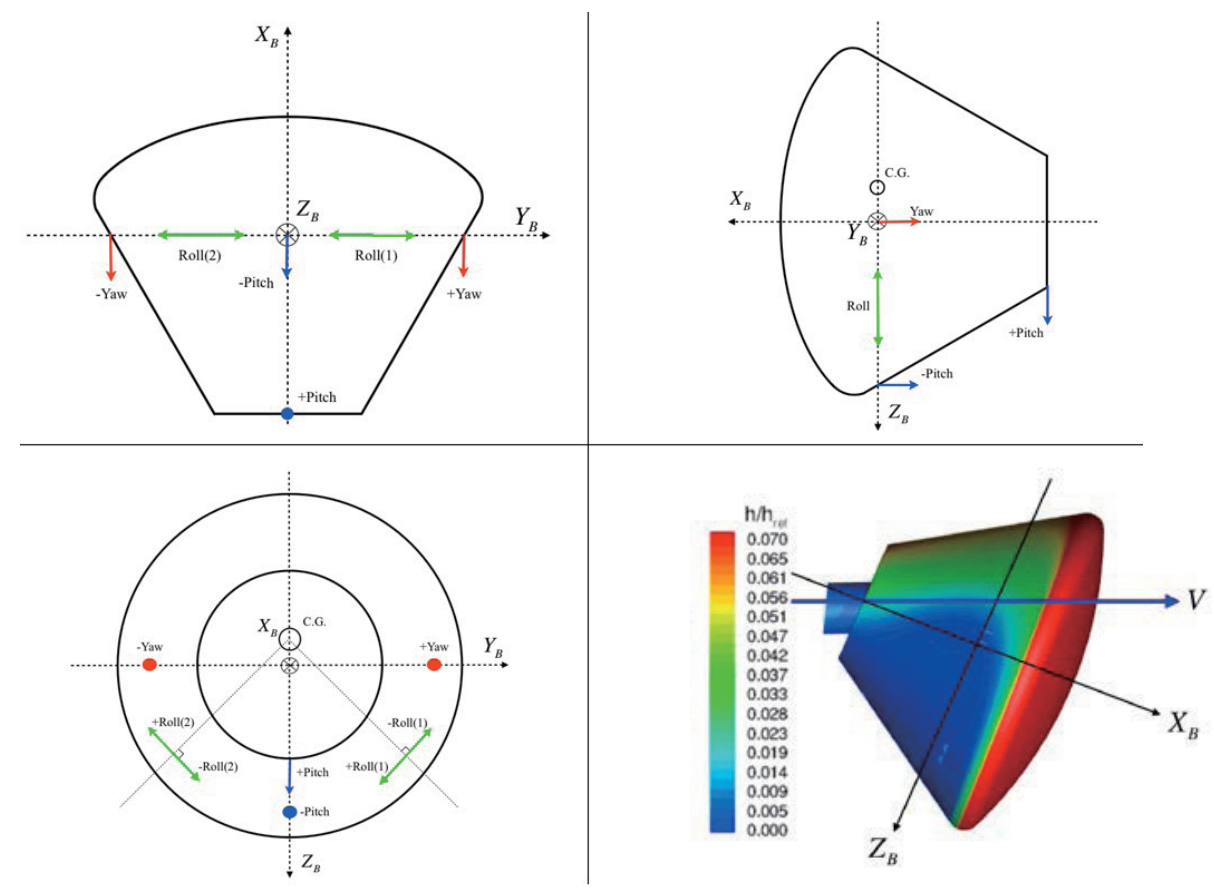

Fig. 1. Vehicle image and RCS location in the present study. (A general example of the heatflux distribution taken from reference ${ }^{4)}$ )

improve the guidance errors by applying combined filtering method of extended Kalman-filter (EKF) and the Sequential Monte-Calro (SMC) on the raw sensor signal with simulated quasi noise.

\section{Trajectory and Attitude Motion Analysis}

\subsection{Vehicle specification and RCS thrusters}

Specifications of the manned space return vehicle now under investigation in JAXA are shown in Table-1 and its outlined shape and the location of the reaction control systems (RCS) is shown in Fig. 1. In the present analysis, the aerodynamic coefficients of the vehicle is calculated based on the modified-Newtonian method, which is enough for the analysis in the hypersonic region, while not in the subsonic region. As will be shown in the latter section, this simplification is considered to be acceptable because the maneuverability of the flight trajectory in the subsonic region is not large in comparison with in the hypersonic

Table 1. Vehicle configuration for analysis .

\begin{tabular}{|c|c|c|}
\hline \multicolumn{2}{|c|}{ Front Area $\left[\mathrm{m}^{2}\right]$} & 4.0 \\
\hline \multicolumn{2}{|c|}{ Diameter $[\mathrm{m}]$} & 4.0 \\
\hline \multicolumn{2}{|c|}{ Length [m] } & 3.2 \\
\hline \multicolumn{2}{|c|}{ Nose Radius [m] } & 4.2 \\
\hline \multicolumn{2}{|c|}{ Vehicle Mass [kg] } & 4000 \\
\hline \multicolumn{2}{|c|}{ Center of Gravity [m] } & {$[0.9167,0.0,-0.110]$} \\
\hline \multicolumn{2}{|c|}{ Aerodynamic Center [m] } & {$[1.4488,0.0,0.0]$} \\
\hline \multicolumn{2}{|c|}{ Trim Angle [deg] } & -19.7 \\
\hline \multicolumn{2}{|c|}{ L/D@trim angle } & 0.3 \\
\hline \multirow{3}{*}{$\begin{array}{l}\text { Moment of Inertia } \\
{[\mathrm{kg}-\mathrm{m} 2]}\end{array}$} & $\mathrm{X}$ & 5110.0 \\
\hline & $\mathrm{Y}$ & 4010.0 \\
\hline & $\mathrm{Z}$ & 3900.0 \\
\hline
\end{tabular}

region. The $\mathrm{L} / \mathrm{D}$ is 0.3 at the trim angle of $20^{\circ}$; the trim angle of $20^{\circ}$ is achieved by the $5 \%$ offset of the gravity center position in the diameter direction as shown in Fig.2.

If the the bank-control thrusters be located symmetrically around the trim axis, they would realize ideal bank-control without generating disturbance forces around the pitch axis nor the yaw. Nevertheless, the bank-control thrusters can not be located symmetrically around the trim axis because they must avoid aerodynamic heating on the
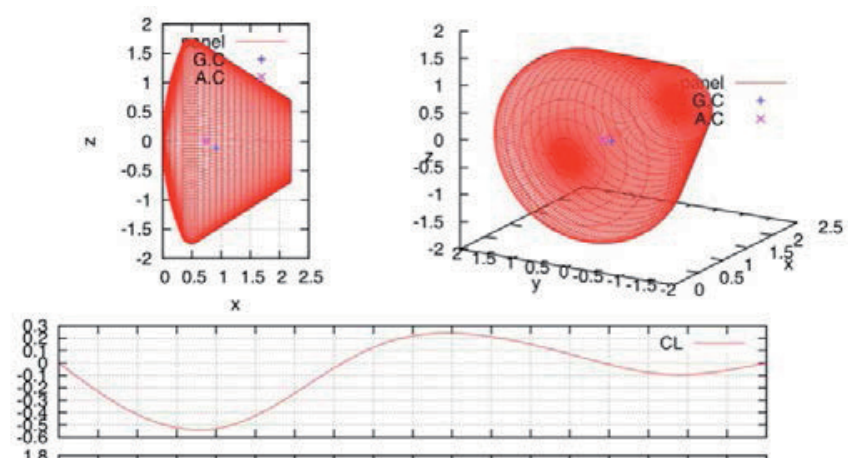

¿

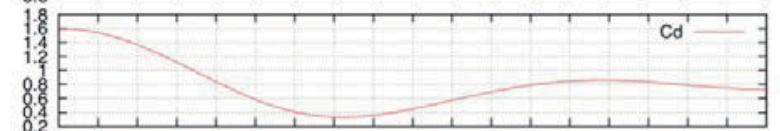

s

छ

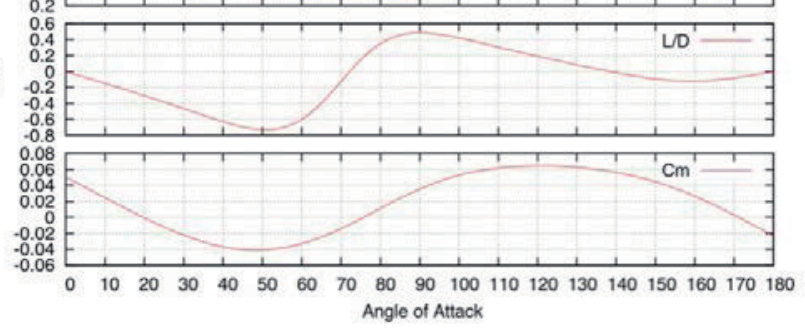

Fig. 2. Aerodynamic characteristics of the baseline model. 
aft-body in case of trimmed attitude as shown in Fig. 1. Taking account of the aerodynamic heating on the aft-body surface, the thrusters are mounted in the shaded/wake region with low aerodynamic heating. Then, the bank-control forces will inevitably generate disturbance forces around the pitch and the yaw axis; which effect is investigated and discussed based on the 6 degrees of freedom (DoF) analysis in the latter section.

In order to generate the angular velocity enough large in short moment, here adapted for the bank-control is reliable on/off pulse control by means of RCS with MMH/NTO propellant: The control force profile used here is simply modeled by simple impulse profile with specific impulse of $330 \mathrm{sec}$ in vacuum and with the minimum impulse of $100 \mathrm{~ms}$ containing transient phase.

\subsection{Nominal reentry trajectory}

The Atmospheric Reentry Analysis (ARENA) code is an integrated flight trajectory and attitude motion analysis code $^{3)}$, which has been used for the flight and landing point analysis of Hayabusa sample return capsule, and orbital survivability analyses of the scientific satellite ever. Though ARENA had been originally written in Fortran, it was redesigned and re-written in $\mathrm{C}++$ program language so as to improve extensibility by adding easily extra modules. The flight analysis was, then, carried out by adding RCS model module to ARENA code.

As for the atmospheric model, 1976 US standard model was adapted for the sake of general discussion. The mass properties of the present return vehicles was already shown in Table 1.

The time profile of the nominal trajectory targeted in the present study for RCS and attitude motion study is plotted in Fig. 3.

\section{RCS Bank-control Characteristics}

\subsection{Bank controll characteristics}

Space return vehicles fly broad region of dynamic pressure and the most efficient control region is considered to be in the vicinity of the maximum dynamic pressure. The control logic around bank and pitch axis is schematically shown in Fig. 4. The bank thruster is fired according to the firing region as designated in bank rate vs. bank error phase plane:

$$
\begin{aligned}
& \sigma_{e}=\sigma-\sigma_{c m d} \\
& \sigma_{e}= \pm \sigma_{D B} \pm(1 / 2 \ddot{\sigma}) \dot{\sigma}^{2}
\end{aligned}
$$

where

$$
\begin{aligned}
& \sigma \text { : Bank Angle, } \dot{\sigma} \text { : Bank Rate, } \\
& \sigma_{\text {cmd }} \text { : Bank Command, } \sigma_{e} \text { : Bank Error, }
\end{aligned}
$$

and

$\sigma_{D B}$ : Bank Angle Dead Band.

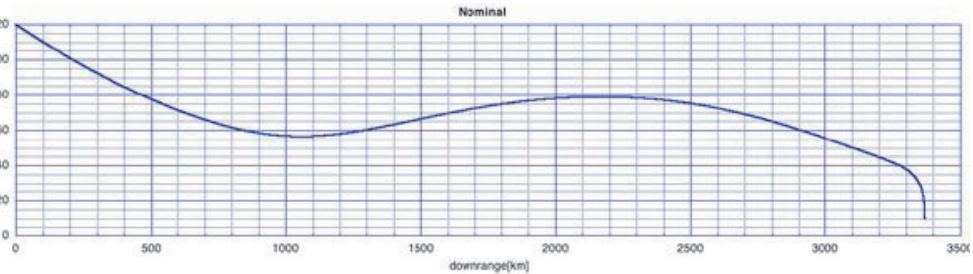

a) Altitude vs. Down Range Characteristics

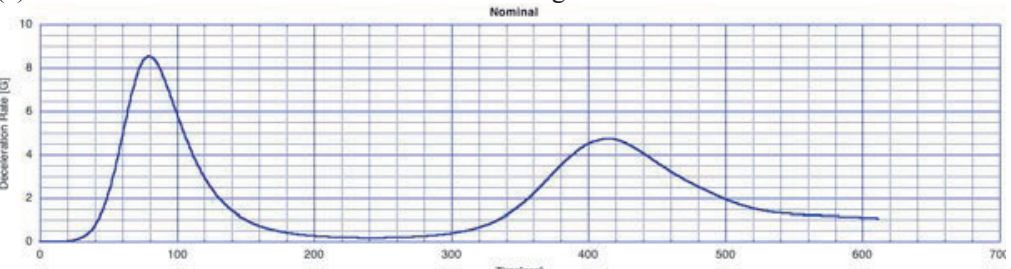

(b) Deceleration Rate [G] vs. Time Profile

ig. 3. Profiles of the altitude, deceleration (root sam of the square of deceleration 3 axises) in the nominal reentry trajectory.

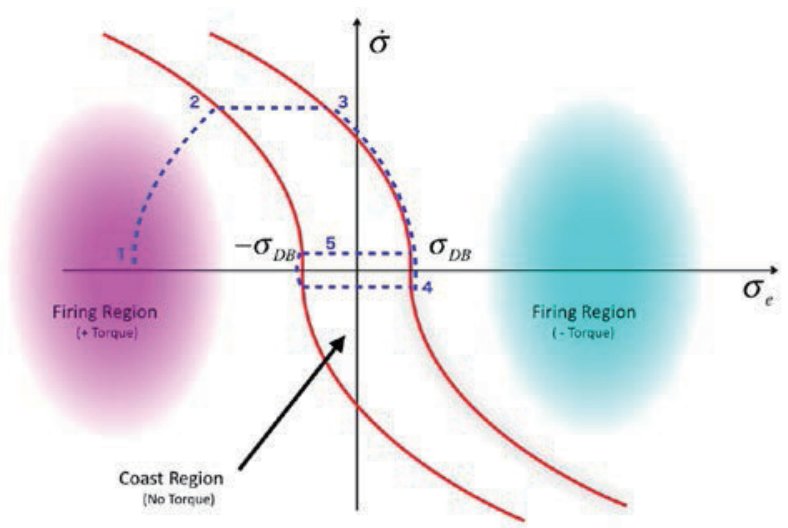

(a) Bank control logic

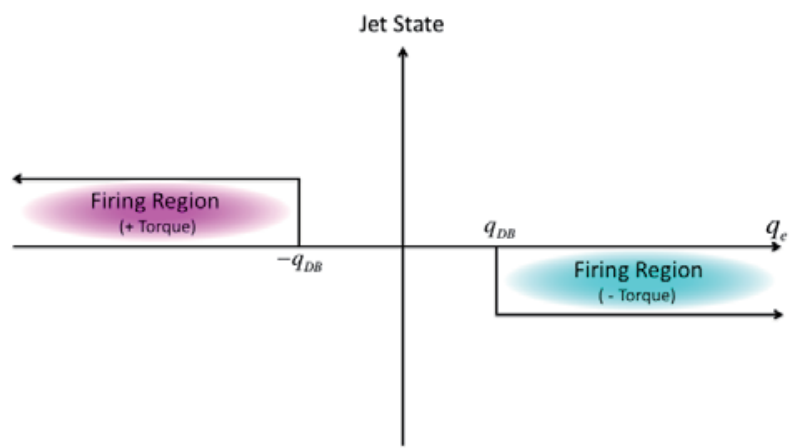

(b) Pitch control logic

Fig. 4. Bank and pitch control logic and dead-band characteristics in the phase plain.

The pitch control thruster (and yaw thruster) is to be fired when the error (designated by present value minus target value) exceeds dead-band region $\mathrm{q}_{\mathrm{DB}}$, respectively as shown in Fig. 4 (b). Control errors between the present value and the ideal are to be neglected as long as the present status is within the dead-band region.

Control characteristics of the bank angle have been investigated with parameters of RCS force ranging from $200 \mathrm{~N}$ to $800 \mathrm{~N}$. An example of the controlled results, time profiles of the bank angle and the rate are shown in Fig. 5 


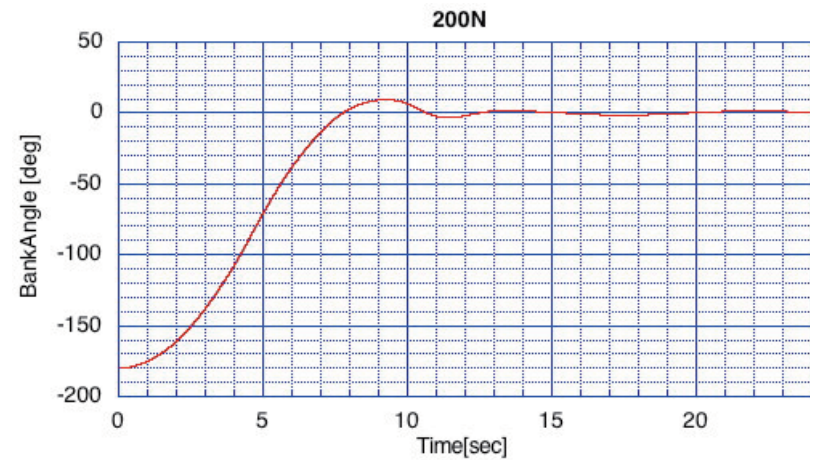

(a) Bank angle converged to the target [200N RCS]

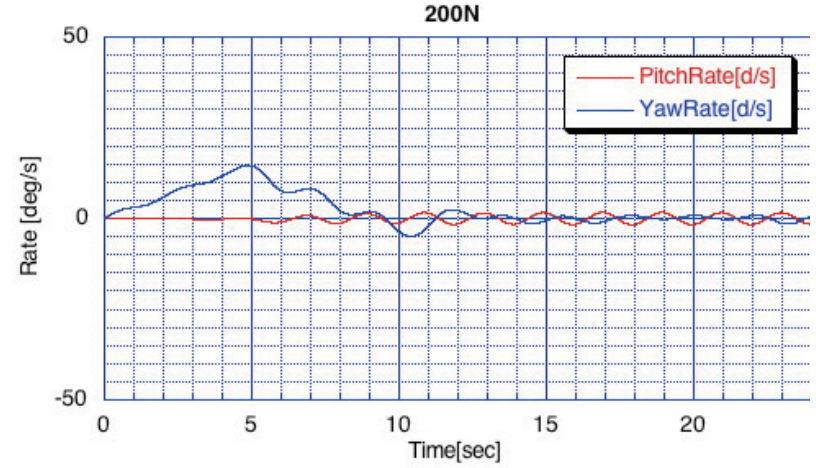

(b) Pitch rate and yaw rate $[200 \mathrm{~N}$ RCS $]$

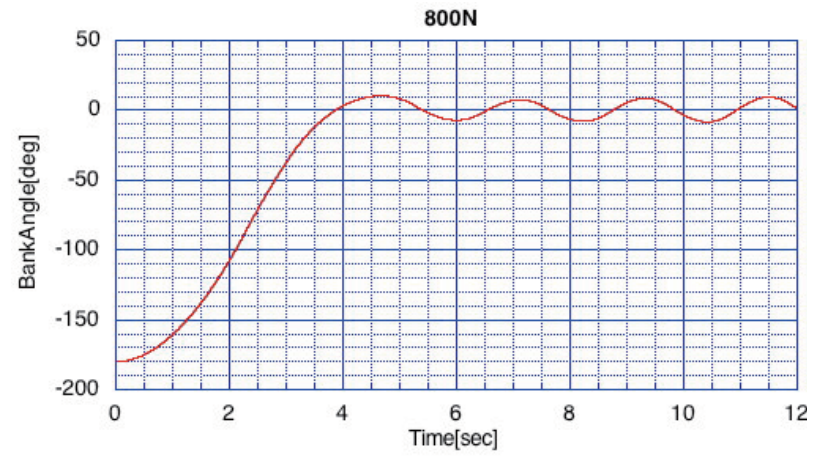

(c) Bank angle converged to the target [800N RCS]

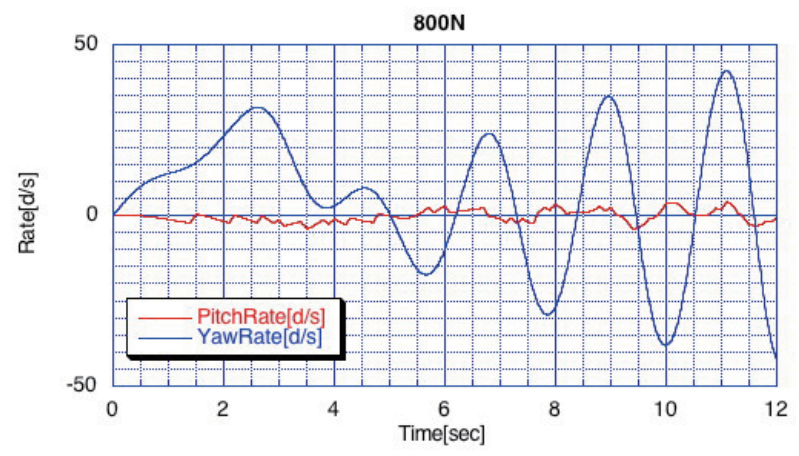

(d) Pitch tate and yaw rate $[800 \mathrm{~N}$ RCS $]$

Fig. 5. Bank angle, pitch/yaw rate characteristics [(a),(b) : $200 \mathrm{~N}$ thruster, (c), (d) $: 800 \mathrm{~N}$ thruster].

with parameters of control force of $200 \mathrm{~N}$ and $800 \mathrm{~N}$ RCS thrusters, initial bank-angle of $0^{\circ}$ and target angle of $180^{\circ}$, respectively. In each case the dead-band is set to be $\pm 2^{\circ}$ around the target value.

In case that the control force exceeds the limitation determined by the minimum impulse and the moment of inertia, the control overshoots as shown in the $800 \mathrm{~N} \mathrm{RCS}$ characteristics as shown in Fig. 5(c). This is because large force RCS fails to control within minimum bank-angle expressed as

$$
\sigma_{\text {min }}=2 \frac{M}{I} \cdot t^{2}
$$

where M : Moment of the control force, I : Moment of Inertia, and $\mathrm{t}:$ minimum impulse, respectively.

Figure $6 \mathrm{~A}$ and $6 \mathrm{~B}$ show the frequency of RCS firing duty during the control process in case of $200 \mathrm{~N}$ RCS and $800 \mathrm{~N}$, respectively. It is recognized that the control firing duty cycles of the $800 \mathrm{~N}$ thruster is more frequent than that of $200 \mathrm{~N}$. In the preliminary study, it was also indicated that about $80 \%$ of the total propellant consumption for the attitude control is attributed to the roll control. In case that the bank control force is excessively large with larger minimum bank-angle, the propellant consumption tends to increase because the number of impulse and frequency is increased due to inability of the appropriate response within the dead-band.

\subsection{Pitch and yaw control characteristics}

Leakage of the control force around the trim axis to the pitch and yaw axis is inevitable because the bank-thrusters cannot be located completely symmetrically due to several limitations such as aerodynamic issues. The pitch control disturbance caused by the leakage of the roll control force is not so severe because of the aerodynamic stability in the

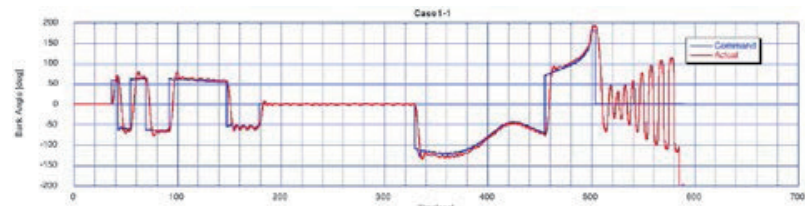

(a) Bank profile

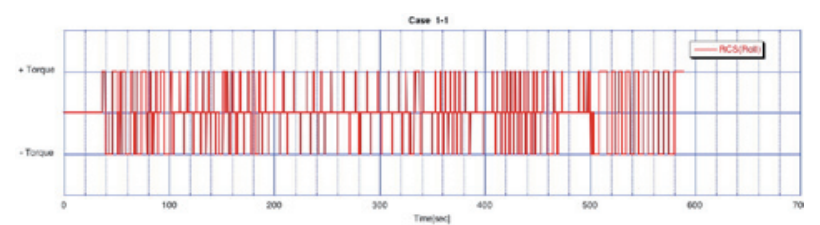

(b) Bank-control profile

Fig. 6A. Bank control by $200 \mathrm{~N}$ roll thruster.

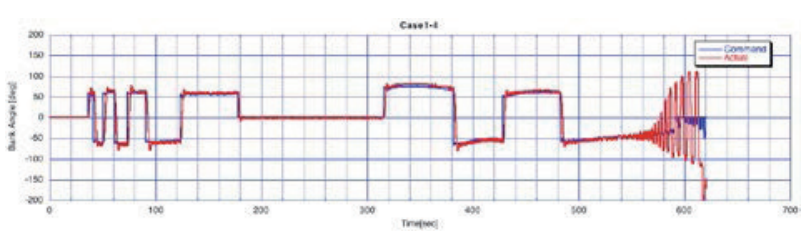

(a) Bank profile

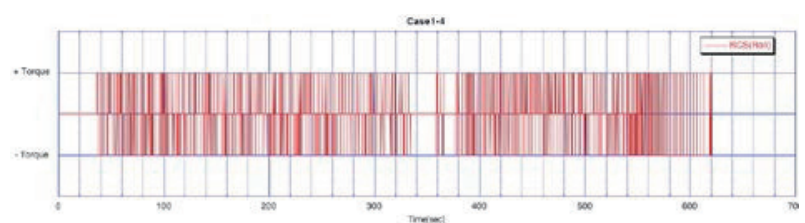

(b) Bank-control profile

Fig. 6B. Bank control by $800 \mathrm{~N}$ roll thruster. 
vicinity of the trim angle, while the yaw control disturbance is much severe because of its weak aerodynamic stability around zero-yawing angle as shown in Figs. 5(b) and 5(d).

\section{Fault Tolerance Algorithm}

One of the significant feature of the guidance and control of the manned space vehicle is the fault tolerance. Historically, two-fault tolerance (2FT) to protect against loss of the crew has been taken for granted in human space flight vehicle design. From a navigation perspective, one of the most robust means of achieving this goal is to compare the outputs of several independent, dissimilar, but equally performing sensors and then use a center select algorithm. This design can tolerate two simultaneous or systematic failures, including common cause failures, and thus fault detection, isolation and recovery of functions all occur automatically with no delay or disruption.

The guidance and control system needs to estimate status properly even in case of the sensor malfunctions. Here we have investigated an example of fault tolerance algorithm by means of Extended Kalman-filter (EKF) and Sequential Monte-Carlo (SMC) method. In the present study, we have investigate state estimation characteristics in a case of the failure that the GPS signal is completely lost during the reentry in the GPS/IMU integrated navigation system, where vehicle flight positioning is to be done by integrating IMU signal without using GPS signal.

\subsection{Extended kalman-filter}

We have designed a state estimation algorithm based on EKF method because nonlinear processes are included in the flight control. Because IMU signals will contain not a small amount of noises, numerically-simulated white noise have been added to the true value. Systematic offset from the true value is not assumed here in the present study.

Figure 7 shows a schematic view of the bank-control input along the entry trajectory and the correspondent bank angle is tabulated in Table-2, where the bank angle is expressed as positive in clockwise direction from the vertical top around trim axis seen from the nose front.

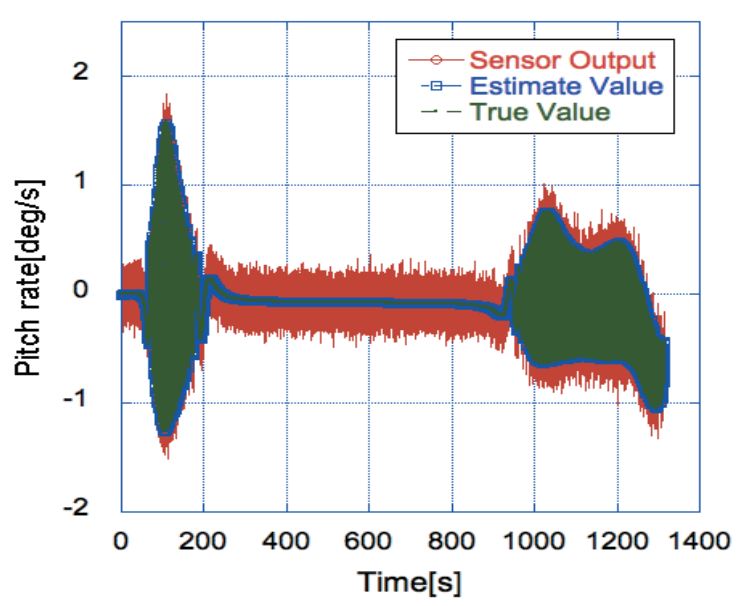

(a) Estimation of the roll rate
EKF calculation step is $10.0 \mathrm{~Hz}$ and uniform noise of $0.1 \mathrm{deg} / \mathrm{s}$ is assumed as white noise. As shown in Fig. 8, EKF state estimation algorithm successfully estimated the true value out of noisy IMU output.

In order to analyze the nonlinear motion of the reentering vehicles, the Extended Kalman-filter (EKF) has been constructed for the continuous variable system. The present study developed an EKF method by using IMU signal solely, which predicts external perturbations exerted on the vehicles during its flight motion strongly affected by the atmosphere, the gravity etc: The EKF estimates the

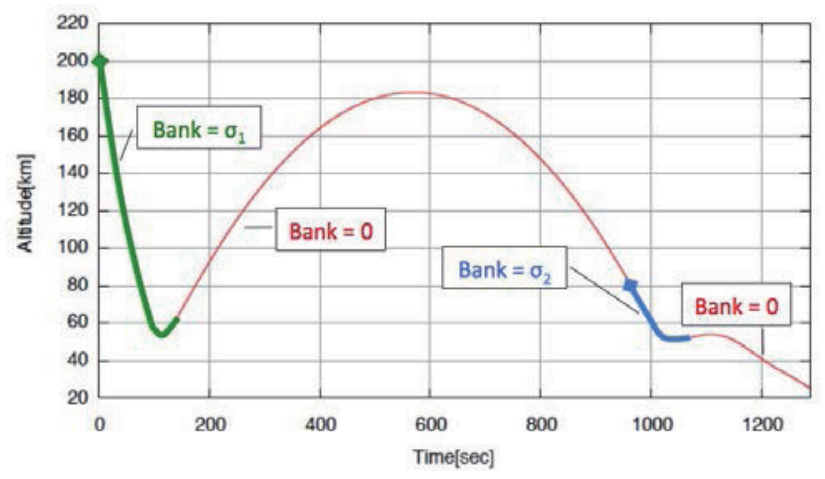

Fig. 7. Schematic view of the bank control input along the entry trajectory.

Table 2. Nominal bank control input.

\begin{tabular}{|c|c|c|c|}
\hline Phase & Control Input & Input $\sigma{ }^{\circ}{ }^{\circ}$ & Altitude \\
\hline \multirow{3}{*}{ 1st Entry } & $\sigma_{1}:$ Bank Angle & $\begin{array}{c}200 \mathrm{~km} \\
\text { (constant) }\end{array}$ \\
\cline { 2 - 4 } & $\begin{array}{c}\text { constant Bank } \\
\text { Control }\end{array}$ & $\begin{array}{c}0.0 \\
\text { (constant) }\end{array}$ & 57.2 \\
\hline \multirow{2}{*}{ 2nd Entry } & $\sigma_{2}:$ Bank Angle & -10 & $\begin{array}{c}80 \\
\text { (constant) }\end{array}$ \\
\cline { 2 - 4 } & $\begin{array}{c}\text { constant Bank } \\
\text { Control }\end{array}$ & $\begin{array}{c}0.0 \\
\text { (constant) }\end{array}$ & 52.9 \\
\hline \multicolumn{2}{|l}{$\begin{array}{l}\text { bank angle is expressed as positive in clockwise } \\
\text { direction from the vertical top around trim axis seen } \\
\text { from the front. }\end{array}$}
\end{tabular}

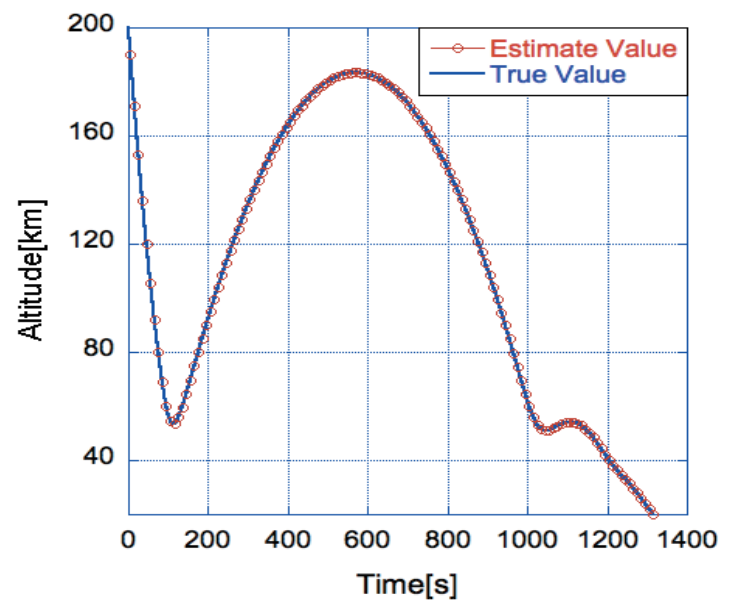

(b) Estimation of the altitude

Fig. 8. EKF estimation example based on the IMU data in case that GPS signals were lost. 


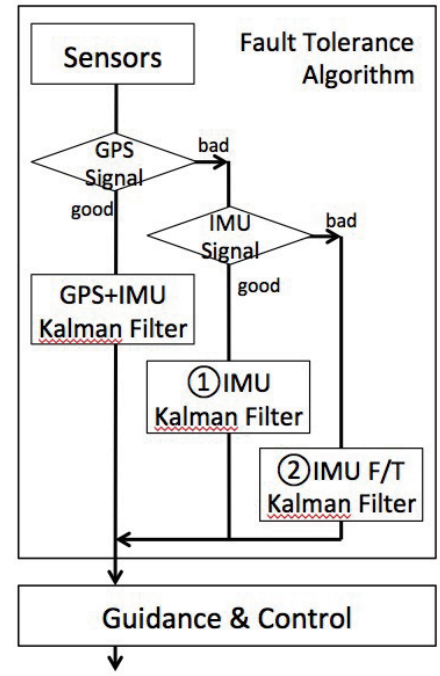

Fig. 9. Fault tolerance algorithm

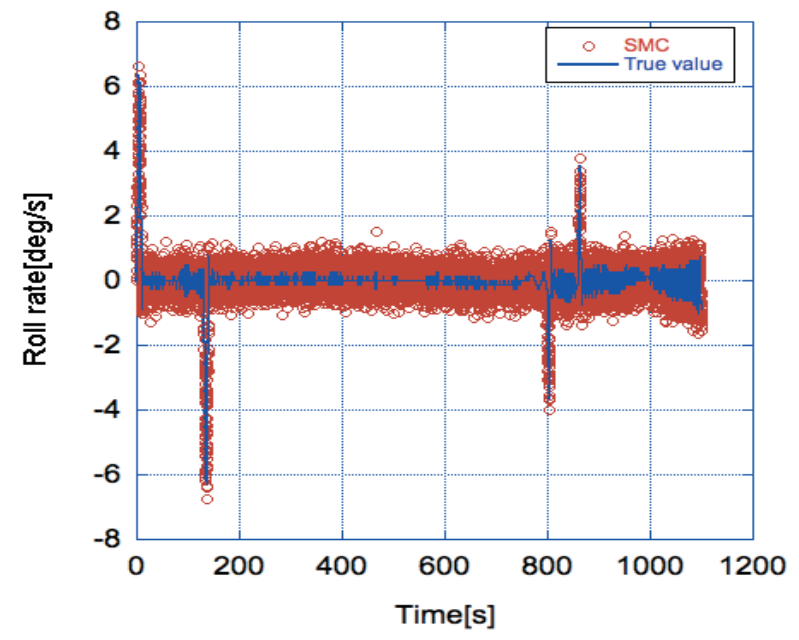

(a) Roll rate (Time : 0 - $1200 \mathrm{sec}$ )

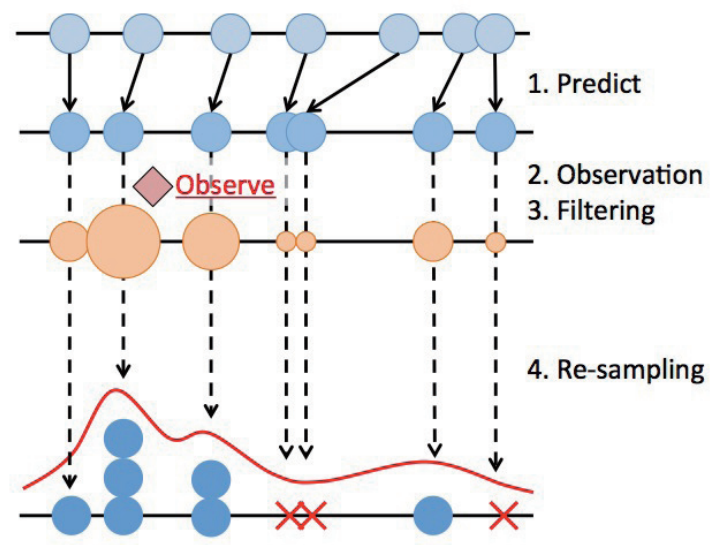

Fig. 10. Schematic view of the sequential Monte-Carlo method for fault tolerance algorithm.

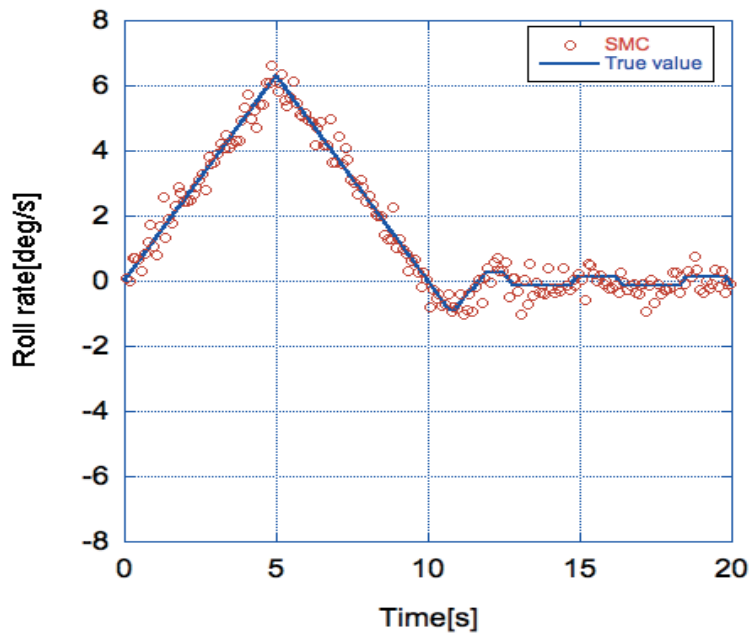

(b) Roll rate in time-magnified scale (Time : 0 - $20 \mathrm{sec}$ )

Fig. 11. Comparison between simulated true value of the roll rate and the SMC estimation.

attitude rotational motion rates and the translational accelerations, in order. The rotational motions and translational motion, in order, constitute the its own system matrix and observation matrix, respectively. The system matrix for the rotational motions in the body-fixed coordinate system is shown as follows;

$$
\begin{aligned}
& x=\left[\begin{array}{ccc}
p & q & r
\end{array}\right]^{T} \\
& A=\left[\begin{array}{ccc}
0 & \omega_{z}\left(I_{y y}-I_{z z}\right) / I_{x x} & \omega_{y}\left(I_{x x}-I_{y y}\right) / I_{z z} \\
\omega_{z}\left(I_{y y}-I_{z z}\right) / I_{x x} & 0 & \omega_{x}\left(I_{z z}-I_{x x}\right) / I_{y y} \\
\omega_{y}\left(I_{x x}-I_{y y}\right) / I_{z z} & \omega_{x}\left(I_{z z}-I_{x x}\right) / I_{y y} & 0
\end{array}\right] \\
& B=\left[\begin{array}{ccc}
-1 / I_{x x} & 0 & 0 \\
0 & -1 / I_{y y} & 0 \\
0 & 0 & -1 / I_{z z}
\end{array}\right] \quad H=\left[\begin{array}{ccc}
1 & 0 & 0 \\
0 & 1 & 0 \\
0 & 0 & 1
\end{array}\right]
\end{aligned}
$$

where $\mathrm{A}, \mathrm{B}$ : system matrix, and $\mathrm{H}$ : the observation matrix of sensors.
The state value in the next step are estimated based on the former step value as shown in the discretized expression of the system :

$$
\begin{aligned}
& x_{k}=\Phi_{k-1} x_{k-1}+B_{k-1} w_{k-1}, \\
& \text { where } \Phi_{k-1}=I+A_{k-1} \Delta, \\
& w_{k-1} \text { : observation errors, } \Delta \text { : calculation step divid. }
\end{aligned}
$$

\subsection{Failure diagnosis and SMC}

In the previous section, the case that IMU signals are all normal and can be integrated to estimate vehicle position. Here considered is a failure case that one of the IMU signal, the roll rate signal for an example, is lost in IMU/GPS integrated navigation system. An example of the fault tolerance algorithm designed in the present study is outlined in Fig. 9; Normally, the flight state parameters are sensed and estimated by means of GPS/IMU integrated navigation system. In case that the GPS signals are lost and IMU signals are still normal, EKF is to be applied to state 
estimation based on IMU as described in the previous section. Moreover, in case that one of the IMU signal, the roll rate, is lost or apparently abnormal, in order to complement the lost roll rate signal, we have adopted Sequential Monte Carlo (SMC) method. The principle of the SMC is schematically shown in Fig. 10: Because each state parameter has relations to each other and one parameter should necessarily affect on other parameters. It is not likely that one parameter is independently changed without leaving other parameters unchanged; Because the $\mathrm{L} / \mathrm{D}$ is a function of bank-angle, which is a function of the integrated value from the roll (bank) rate, and the L/D characteristics affects on the acceleration, thus, there should be a strong relation between the roll rate and accelerometer output. SMC utilizes these relations, and estimated most probable states by generating probable values of the lost data, observing their effect on other parameters, and finally selecting/adopting the most 'reasonable' combination set of the lost values.

Sequential Monte Carlo (SMC) or Particle filter methods are a set of on-line posterior density estimation algorithms that estimate the maximum likelihood posterior density of the state-space by directly implementing the Bayesian recursion equations, which utilizes statistically the density distribution of the particles which represent the state values ${ }^{5,6)}$.

The samples from the distribution are represented by a set of particles; each particle has a weight assigned to it that represents the probability of that particle being sampled from the probability density function.

Weight disparity leading to weight collapse is a common issue encountered in these filtering algorithms; however it can be mitigated by including a resampling step before the weights become too uneven. In the resampling step, the particles with negligible weights are replaced by new particles in the proximity of the particles with higher weights.

Let the average of the sample set complied with the prior probability distribution be

$$
S_{t \mid t-1}=\left\{S_{t \mid t-1}^{(1)}, S_{t \mid t-1}^{(2)}, \ldots . S_{t \mid t-1}^{(N)}\right\}
$$

and with the posterior probability distribution

$$
S_{t \mid t}=\left\{S_{t \mid t}^{(1)}, S_{t \mid t}^{(2)}, \ldots . S_{t \mid t}^{(N)}\right\}
$$

The prior $p\left(x_{t} \mid y_{t-1}\right)$ and posterior $p\left(x_{t} \mid y_{t}\right)$ distributions are expressed as follows, respectively.

$$
\begin{aligned}
& p\left(x_{t} \mid y_{t-1}\right) \approx \frac{1}{N} \sum_{i=1}^{N} \delta\left(x_{t}-S_{t \mid t-1}^{(i)}\right) \\
& p\left(x_{t} \mid y_{t}\right) \approx \frac{1}{N} \sum_{i=1}^{N} \delta\left(x_{t}-S_{t \mid t}^{(i)}\right)
\end{aligned}
$$

where $\delta$ is the Dirac's delta function.

In the SMC, the two sample sets, $S_{t \mid t-1}$ and $S_{t \mid t}$ are generated based on the prior and the posterior probability distributions. According to the Bavesian theorem. these two

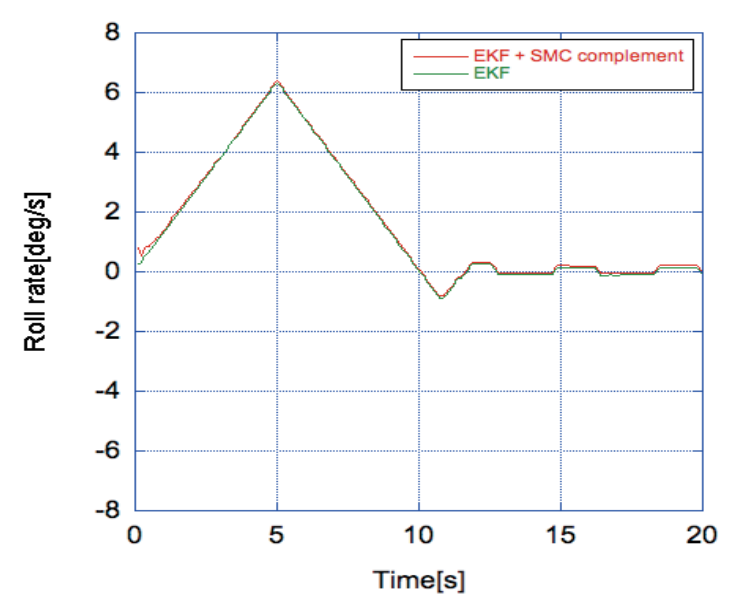

Fig. 12. Comparison between simulated true value of the roll rate and the EKF+SMC estimation.

distributions are replaced by the product of the probability and each likelihood.

In the present study, the maximum likelihood states are to be estimated in the situation where the sensor output signal of the roll angle is lost. The accelerometer sensor output values which considered to be normal are used to evaluate a likelihood function.

$$
\begin{aligned}
& S=\left(A c c_{e s t}-A c c_{o b s}\right)^{T}\left(A c c_{e s t}-A c c_{o b s}\right) \\
& A c c_{e s t} \text { : estimated acceleration vector, } \\
& A c c_{o b s} \text { : observed acceleration vector. }
\end{aligned}
$$

Figure 11 shows a comparison between simulated true value of the roll rate and the SMC estimation. From the Fig. 11(b) of the roll rate in the time-magnified scale, it is recognized that SMC successfully estimated roll rate from other state parameters even in case that it is completely lost. Moreover the EKF has been applied to the above SMC estimation, and we have obtained good agreement between the true value and the state estimation (roll rate) by EKF + SMC method as shown in Fig. 12.

\section{Conclusion}

A capsule-type vehicle is promising for the manned return vehicle from the Moon, Mars or from further bodies from the stand point of the weight budget regardless of its drawback of relatively low lift-to-drag (L/D) aerodynamic characteristics. Vehicle flight control at the skip entry needs to be carried out through bank-control by reaction control systems (RCS) which is forced to be asymmetrically arranged due to the aft-body aerodynamic heating. The asymmetrical arrangement causes the undesirable attitude motion of the vehicle, especially in yawing motion. Bank control errors tend to decrease the L/D control efficiency and may lead to errors in guidance in landing point. The present paper describes the 6 degree of freedom (6DoF) simulation of the skip entry of the 4-ton class vehicle and 
discussed the related issues. Effect of the signal quality (noise) and errors of the bank-angle and the rate sensors are investigated qualitatively and the guidance errors have been improved by applying combined filtering method of Kalman-filter and the Sequential Monte-Calro on the raw sensor signal with simulated quasi noise.

We have investigated the effect of the RCS control forces and the thrust vector direction errors on the bankcontrol characteristics and on the amount of the propellant. While the rapid bank-control response is achieved around the trim axis by the larger control force, the accuracy is deteriorated by its larger minimum control angle determined according to the force itself, which consequently deteriorates control performance and propellant consumption efficiency.

Leakage of the control force around the trim axis to the pitch and yaw axis is inevitable because the bank-thrusters cannot be located completely symmetrically due to several limitations such as aerodynamic issues. The pitch control disturbance caused by the leakage of the roll control force is not so severe because of the aerodynamic stability in the vicinity of the trim angle, while the yaw control disturbance is much severe because of its weak aerodynamic stability around zero-yawing angle.

A fault tolerance algorithm based on Extended Kalmanfilter (EKF) and Sequential Monte-Carlo (SMC) method was designed and its application validity was investigated. Under the assumption of using the GPS/IMU integrated in a case of the failure that the GPS signal is completely lost during the reentry, numerical simulation has showed that $\mathrm{EKF}+\mathrm{SMC}$ fault tolerance algorithm successfully achieved state estimation of vehicle flight positioning by integrating IMU signal even under the condition that the roll rate and GPS signals are lost.

\section{Acknowledgements}

We are highly indebted to Mr. Yusuke Kurihara for his great effort in the present research. We also would like to express our gratitude towards his supervising associate professor, Dr. Sahara in Tokyo Metropolitan University for his kind co-operation and understanding.

\section{References}

1) Yamada, T., Inatani, Y. et. al. : Post-Flight Analysis of the Recovered Hayabusa Heatshield and Results from Airborne Observation, 61st International Astronautical Congress, Praha, Naples, Italy, IAC-12-C2-1-9-x15178, Oct., 2012.

2) Yoshihara, K. and Yamada, T. : Post-Flight Analysis of the Recovered Hayabusa Heatshield and Results from Airborne Observation, submitted to 29th ISTS, Nagoya.

3) Yamada, T. : 'ARENA : Atmospheric Reentry Analysis Code ', 1H04, Proc. 56th JSASS Space Science and Tech. Beppu, Japan, 2012.

4) Dyakonov, A. A., Buck, G. M. and Decaro, A. D. : Analysis of Aeroheating Augmentation due to Reaction Control System Jets on Orion Crew Exploration Vehicle", AIAA Thermophysics Conference 41st, San Antonia, TX, 22-25 June 2005.

5) Doucet, A., Fretas, N. and Gordon, N. : Sequential Monte Carlo Methods in Practice, Springer, ISBN-10 0387951466, 2001.

6) Kato, T. : Implementation of the Particle Filter, 2007 Report, Information Processing Society of Japan, 2007. 